\title{
Polarized and homogeneous embeddings of dual polar spaces
}

\author{
R.J. Blok • I. Cardinali • B. De Bruyn • A. Pasini
}

Received: 12 May 2008 / Accepted: 22 December 2008 / Published online: 13 January 2009

(C) Springer Science+Business Media, LLC 2008

\begin{abstract}
Let $\Gamma$ be the dual of a classical polar space and let $e$ be a projective embedding of $\Gamma$, defined over a commutative division ring. We shall prove that, if $e$ is homogeneous, then it is polarized.
\end{abstract}

Keywords Dual polar spaces $\cdot$ Polarized embeddings $\cdot$ Homogeneous embeddings

\section{Introduction}

1.1 The main result of this paper

In the sequel we assume that the reader is familiar with dual polar spaces and projective embeddings. If not, we refer him to Section 2, where all definitions and basic results to be used in this paper are exposed. In this introduction we shall only briefly explain a couple of notions which occur in the statement of our main result.

R.J. Blok (凶)

Department of Mathematics and Statistics, Bowling Green State University, Bowling Green, OH 43403, USA

e-mail: blokr@member.ams.org

I. Cardinali

Information Engineering Department, University of Siena, Via Roma 56, 53100 Siena, Italy e-mail: cardinali3@unisi.it

B. De Bruyn

Department of Pure Mathematics and Computer Algebra, Ghent University, Krijgslaan 281 (S22), 9000 Gent, Belgium

e-mail: bdb@cage.ugent.be

A. Pasini

Department of Mathematics, University of Siena, Pian dei Mantellini 44, 53100 Siena, Italy

e-mail: pasini@unisi.it 
Let $\Gamma$ be an embeddable thick dual polar space of rank $n \geq 2$. We recall that all embeddings of $\Gamma$ are defined over the same division ring $\mathbb{F}$, called the underlying division ring of $\Gamma$ (see Subsection 2.2). We assume that $\mathbb{F}$ is commutative. We also assume that $\Gamma$ is classical, by which we mean that $\Gamma$ is the dual of an embeddable polar space.

First, we consider a symmetry condition on an embedding $e$ for $\Gamma$. We denote by $\operatorname{Aut}(\Gamma)_{0}$ the normal subgroup of $\operatorname{Aut}(\Gamma)$ generated by the root groups of the building associated to $\Gamma$ (see Subsection 3.4). (The group $\operatorname{Aut}(\Gamma)_{0}$ is in fact the largest normal simple subgroup of $\operatorname{Aut}(\Gamma))$. We say that $e$ is $\operatorname{Aut}(\Gamma)_{0}$-homogeneous if $\operatorname{Aut}(\Gamma)_{0}$ lifts through $e$ to a subgroup of the full automorphism group $\mathrm{P} \Gamma \mathrm{L}(V)$ of $\mathrm{PG}(V)$.

Next, we consider a geometric condition on the embedding $e$. The embedding $e$ is said to be polarized if, for every point $x$ of $\Gamma$, the image $e\left(H_{x}\right)$ of the hyperplane $H_{x}$ of $\Gamma$, formed by the points at non-maximal distance from $x$, spans a hyperplane of $\mathrm{PG}(V)$. The following theorem, which we will prove in Section 4, is the main result of this paper. It exhibits a close connection between the symmetry and geometry of the embedding $e$.

Theorem 1.1 If e is Aut $(\Gamma)_{0}$-homogeneous, then it is polarized.

\subsection{Another formulation of Theorem 1.1}

Let $\Gamma$ be a classical embeddable thick dual polar space of rank $n \geq 2$ defined over a commutative division ring as in Subsection 1.1.

Let $\tilde{e}: \Gamma \rightarrow \operatorname{PG}(\tilde{V})$ be the absolutely universal embedding of $\Gamma$, which exists by Kasikova and Shult [29, 4.6]. As $\tilde{e}$ is absolutely universal, the full automorphism group $\operatorname{Aut}(\Gamma)$ of $\Gamma$ lifts through $\tilde{e}$ to a subgroup of $\operatorname{P\Gamma L}(\widetilde{V})$. Therefore, every subgroup $G$ of $\operatorname{Aut}(\Gamma)$ lifts as well to a subgroup $\tilde{e}(G)$ of $\mathrm{P} \Gamma \mathrm{L}(\tilde{V})$. In particular, $\tilde{e}$ is also $\operatorname{Aut}(\Gamma)_{0}$-homogeneous. Hence it is polarized by Theorem 1.1 (see also Cardinali, De Bruyn and Pasini [11, Corollary 1.8] for a different, more straightforward proof of this claim).

Let us denote the point-set of $\Gamma$ by $\mathcal{P}$. We call $R=\bigcap_{x \in \mathcal{P}}\left\langle\tilde{e}\left(H_{x}\right)\right\rangle$ the nucleus of $\tilde{e}$. By Cardinali, De Bruyn and Pasini [10], $R$ defines a quotient $\tilde{e} / R$ of $\tilde{e}$ which is polarized. Moreover, an embedding of $\Gamma$ is polarized if and only if it admits a (possibly improper) quotient isomorphic to $\tilde{e} / R$. For this reason, $\tilde{e} / R$ is called the minimal polarized embedding of $\tilde{e}$. Note that $R$ might be trivial. If that is the case then $\tilde{e}$ is the unique polarized embedding of $\Gamma$.

Clearly $R$ is an $\operatorname{Aut}(\Gamma)$-invariant subspace of $\operatorname{PG}(\tilde{V})$, where we say that a subspace $U$ of $\operatorname{PG}(\widetilde{V})$ is $G$-invariant for a subgroup $G \leq \operatorname{Aut}(\Gamma)$ if it is stabilized by $\tilde{e}(G)$. As $R$ is $\operatorname{Aut}(\Gamma)$-invariant, it is $G$-invariant for every subgroup $G$ of $\operatorname{Aut}(\Gamma)$. In particular, $R$ is $\operatorname{Aut}(\Gamma)_{0}$-invariant. Hence $\tilde{e} / R$ is $\operatorname{Aut}(\Gamma)_{0}$-homogeneous. As we shall see in Section 4, Theorem 1.1 implies the following:

Theorem 1.2 All Aut $(\Gamma)_{0}$-invariant proper subspaces of $\mathrm{PG}(\tilde{V})$ are contained in $R$.

In other words $R$, regarded as a subspace of $\widetilde{V}$, is the largest proper $Z \cdot \tilde{e}(G)$ submodule of $\widetilde{V}$, where $G=\operatorname{Aut}(\Gamma)_{0}, Z$ stands for the center of $\operatorname{GL}(\widetilde{V})$ and $Z \cdot \tilde{e}(G)$ is the preimage of $\tilde{e}(G)$ by the projection of $\operatorname{GL}(\tilde{V})$ onto $\operatorname{PGL}(\widetilde{V})$. 


\subsection{Some special cases}

A number of consequences can immediately be drawn from Theorems 1.1 and 1.2. We shall gather them in Corollary 1.3, to be stated below, but we first recall some facts.

Let $\mathrm{Q}(2 n, \mathbb{F})$ be the orthogonal polar space of rank $n \geq 2$ of parabolic type over a field $\mathbb{F}$ and let $\Gamma=\mathrm{DQ}(2 n, \mathbb{F})$ be its dual. We have $\operatorname{Aut}(\Gamma)_{0}=\operatorname{P} \Omega(2 n+1, \mathbb{F})$. The dual polar space $\Gamma$ admits a polarized embedding $e_{\text {spin }}: \Gamma \rightarrow \operatorname{PG}\left(2^{n}-1, \mathbb{F}\right)$, called the spin embedding of $\Gamma$ (Buekenhout and Cameron [6, Section 7]; see also De Bruyn [21]). When char $(\mathbb{F}) \neq 2$ the embedding $e_{\text {spin }}$ is universal (Wells [41]). This also follows from the fact that in this case $\Gamma$ admits a generating set of size $2^{n}$ (Blok and Brouwer [2], Cooperstein and Shult [16]). If $\operatorname{char}(\mathbb{F})=2$ and $\mathbb{F}$ is perfect then $\mathrm{Q}(2 n, \mathbb{F})$ is isomorphic to the symplectic polar space $\mathrm{W}(2 n-1, \mathbb{F})$ of rank $n$ over $\mathbb{F}$. Accordingly, $\mathrm{DQ}(2 n, \mathbb{F}) \cong \mathrm{DW}(2 n-1, \mathbb{F})$. The dual polar space DW $(2 n-1, \mathbb{F})$ admits embeddings in projective spaces of dimension larger than $2^{n}-1$, which we will discuss later.

Let $\mathrm{Q}^{-}(2 n+1, \mathbb{F})$ be the orthogonal polar space of elliptic type arising from a nonsingular quadratic form of Witt index $n \geq 2$ over a field $\mathbb{F}$, which becomes a quadratic form of Witt index $n+1$ when regarded over a quadratic Galois extension $\mathbb{F}_{1}$ of $\mathbb{F}$. Let $\Gamma=\mathrm{DQ}^{-}(2 n+1, \mathbb{F})$ be its dual. Then $\operatorname{Aut}(\Gamma)_{0}=\mathrm{P}^{-}(2 n+2, \mathbb{F})$. The dual polar space $\Gamma$ admits a polarized embedding in $\mathrm{PG}\left(2^{n}-1, \mathbb{F}_{1}\right)$, often called the spin embedding of $\Gamma$. It arises from the half-spin embedding of the half-spin geometry of $\mathrm{Q}^{+}\left(2 n+1, \mathbb{F}_{1}\right)$. We refer to Cooperstein and Shult [17] and De Bruyn [22] for details. We shall denote this embedding by the symbol $e_{\text {spin }}^{-}$. The embedding $e_{\text {spin }}^{-}$is universal. Indeed, $\Gamma$ admits a generating set of size $2^{n}$ (Cooperstein and Shult [17] for the finite case, De Bruyn [22] for the general case).

Let $\mathrm{H}\left(2 n-1, \mathbb{F}_{0}^{2}\right)$ be the hermitian polar space associated to a nonsingular hermitian variety of Witt index $n \geq 2$ in $\operatorname{PG}\left(2 n-1, \mathbb{F}_{0}^{2}\right)$. Here, $\mathbb{F}_{0}$ is the subfield of $\mathbb{F}=$ $\mathbb{F}_{0}^{2}$ fixed by the involutory automorphism of $\mathbb{F}$ which defines the hermitian variety. Let $\Gamma=\operatorname{DH}\left(2 n-1, \mathbb{F}_{0}^{2}\right)$ be the dual of $\mathrm{H}\left(2 n-1, \mathbb{F}_{0}^{2}\right)$. We have $\operatorname{Aut}(\Gamma)_{0}=\operatorname{PSU}\left(2 n, \mathbb{F}_{0}^{2}\right)$. The dual polar space $\Gamma$ admits a polarized embedding in $\operatorname{PG}\left(N-1, \mathbb{F}_{0}\right)$, where $N=\left(\begin{array}{c}2 n \\ n\end{array}\right)$. (See Cooperstein [13]; also Cardinali, De Bruyn and Pasini [10], De Bruyn [20].) We call this embedding the Grassmann embedding of $\Gamma$ and we denote it by $e_{\mathrm{gr}}^{H}$. The subscript 'gr' and the superscript $H$ should remind us of the word 'Grassmann' and the fact that $\Gamma$ is of hermitian type, in order to avoid confusion with Grassmann embeddings of dual polar spaces of symplectic type, to be discussed later in this introduction. The attribute 'Grassmann' is motivated by the fact that $e_{\mathrm{gr}}^{H}$ arises from the usual embedding of the grassmannian of $n$-subspaces of $V(2 n, \mathbb{F})$ in $\operatorname{PG}(N-1, \mathbb{F})$, via the choice of a suitable Baer subgeometry $\operatorname{PG}\left(N-1, \mathbb{F}_{0}\right)$ of $\operatorname{PG}(N-1, \mathbb{F})$. If $\left|\mathbb{F}_{0}\right|>2$ then $e_{\mathrm{gr}}^{H}$ is universal. Indeed, in this case $\Gamma$ admits a generating set of size $\left(\begin{array}{c}2 n \\ n\end{array}\right)$ (see Cooperstein [13] for the finite case and De Bruyn and Pasini [25] for the general case). We refer to $\mathrm{Li}[31]$ for information on the universal embedding of $\Gamma$ when $\left|\mathbb{F}_{0}\right|=2$.

Corollary 1.3 (1) Let $\Gamma=\operatorname{DQ}(2 n, \mathbb{F})$ (hence $\operatorname{Aut}(\Gamma)_{0}=\operatorname{P} \Omega(2 n+1, \mathbb{F})$ ). If $\operatorname{char}(\mathbb{F}) \neq 2$ then the spin embedding $e_{\text {spin }}: \Gamma \rightarrow \operatorname{PG}\left(2^{n}-1, \mathbb{F}\right)$ is the unique 
Aut $(\Gamma)_{0}$-homogeneous embedding of $\Gamma$. If char $(\mathbb{F})=2$ then $e_{\text {spin }}$ is the smallest Aut $(\Gamma)_{0}$-homogeneous embedding of $\Gamma$.

(2) Let $\Gamma=\mathrm{DQ}^{-}(2 n+1, \mathbb{F})$ (hence $\left.\operatorname{Aut}(\Gamma)_{0}=\mathrm{P}^{-}(2 n+2, \mathbb{F})\right)$. Then the spin embedding $e_{\text {spin }}^{-}$is the unique Aut $(\Gamma)_{0}$-homogeneous embedding of $\Gamma$.

(3) Let $\Gamma=\operatorname{DH}\left(2 n-1, \mathbb{F}_{0}^{2}\right)$ (hence $\left.\operatorname{Aut}(\Gamma)_{0}=\operatorname{PSU}\left(2 n, \mathbb{F}_{0}^{2}\right)\right)$. If $\left|\mathbb{F}_{0}\right|>2$ then the Grassmann embedding $e_{\mathrm{gr}}^{H}$ is the unique Aut $(\Gamma)_{0}$-homogeneous embedding of $\Gamma$. If $\left|\mathbb{F}_{0}\right|=2$ then $e_{\mathrm{gr}}^{H}$ is the smallest $\operatorname{Aut}(\Gamma)_{0}$-homogeneous embedding of $\Gamma$.

Proof As the proof is very short, we shall give it here. Parts (1) and (2) immediately follow from Theorem 1.1, the fact that every polarized embedding of a dual polar space has projective dimension at least $2^{n}-1$ (De Bruyn and Pasini [26]) and what we have said above on the universal embeddings of $\mathrm{DQ}(2 n, \mathbb{F})$ and $\mathrm{DQ}^{-}(2 n+1, \mathbb{F})$. Part (3) follows from Theorem 1.1, the fact that $e_{\mathrm{gr}}^{H}$ admits no proper polarized quotients (Cardinali, De Bruyn and Pasini [10]) and what we have said above on the universal embedding of $\mathrm{DH}\left(2 n-1, \mathbb{F}_{0}^{2}\right)$.

When $\Gamma=\mathrm{DW}(2 n-1, \mathbb{F})$ (dual of the polar space $\mathrm{W}(2 n-1, \mathbb{F})$ of symplectic type and rank $n \geq 2$ over $\mathbb{F}$ ) then things go quite differently than in the cases considered in Corollary 1.3. The dual polar space $\Gamma$ admits the so-called Grassmann embedding $e_{\mathrm{gr}}: \Gamma \rightarrow \operatorname{PG}(V)$, where $V=V(N, \mathbb{F}), N=\left(\begin{array}{c}2 n \\ n\end{array}\right)-\left(\begin{array}{c}2 n \\ n-2\end{array}\right)$. We refer the reader to Cooperstein [14] (also Cooperstein and Shult [17], Cardinali, De Bruyn and Pasini [10], De Bruyn [19]) for the definition of $e_{\mathrm{gr}}$ and a discussion of its properties. We only recall that $e_{\mathrm{gr}}$ is polarized (Cardinali, De Bruyn and Pasini [10]) and it is universal when $|\mathbb{F}|>2$ (see Cooperstein [14] for the finite case and De Bruyn and Pasini [25] for the infinite case). When $|\mathbb{F}|=2$, the dimension of the universal embedding of $\Gamma$ has been determined by Li [30] (also Blokhuis and Brouwer [5]). In any case, $\operatorname{Aut}(\Gamma)_{0}=\operatorname{PSp}(2 n, \mathbb{F})$.

Let $R$ be the nucleus of $e_{\mathrm{gr}}$, regarded as a subspace of $V=V(N, \mathbb{F})$. If $\operatorname{char}(\mathbb{F})=0$ then $R=0$ (see e.g. De Bruyn [24]). On the other hand, when $\operatorname{char}(\mathbb{F}) \neq 0$ then in general $R \neq 0$. In case char $(\mathbb{F})>2$ a recursive formula by Premet and Suprunenko in [33] describes the dimensions of the modules in the decomposition series of $R$ as a module for $\operatorname{PSp}(2 n, \mathbb{F})$.

Let us consider the case char $(\mathbb{F})=2$. By Blok, Cardinali and De Bruyn [3] (see also Cardinali and Lunardon [12] for the rank 3 case), if char $(\mathbb{F})=2$ then $R$ has vector dimension $\operatorname{dim}(R)=N-2^{n}$. In this case $e_{\mathrm{gr}} / R\left(\right.$ which is the minimal $\operatorname{Aut}(\Gamma)_{0^{-}}$ homogeneous embedding of $\Gamma$, by Theorem 1.1) has projective dimension $2^{n}-1$. In particular, if $\mathbb{F}$ is perfect then $\Gamma \cong \mathrm{DQ}(2 n, \mathbb{F})$ and $e_{\mathrm{gr}} / R$ is just the spin embedding of DQ $(2 n, \mathbb{F})$. An analysis similar to that of Premet and Suprunenko in the case $\operatorname{char}(\mathbb{F})=2$ was done by Adamovich [1]. The results by Premet, Suprunenko and Adamovich are rather algebraic in nature. The present paper, along with $[3,10]$ is the result of an initiative by Blok, Cardinali and Pasini to provide a more geometric insight into their results.

We conclude this section with a remark on some irreducibility consequences to our main results. From Claim (3) of Corollary 1.3 one can readily deduce (see Lemma 4.3 of Section 4.2) that the $\left(\begin{array}{c}2 n \\ n\end{array}\right)$-dimensional module for PSU $\left(2 n, \mathbb{F}_{0}^{2}\right)$ produced by the Grassmann embedding $e_{\mathrm{gr}}^{H}$ of $\mathrm{DH}\left(2 n-1, \mathbb{F}_{0}^{2}\right)$ is irreducible. This latter fact is also 
mentioned by Cooperstein [13, proof of Proposition 5.1], but with no proof. A proof, different from the one we have given above, is implicit in Blok and Cooperstein [4]. One more proof, based on linear algebra, has been found by De Bruyn [24]. A proof of Theorem 1.2 is also included in [24] for the case $\tilde{e}$ is the Grassmann embedding of either $\mathrm{DW}(2 n-1, \mathbb{F})$ or $\mathrm{DH}\left(2 n-1, \mathbb{F}_{0}^{2}\right)$.

\subsection{Organization of the paper}

In Section 2 we recall definitions and basics on point-line geometries, dual polar spaces and embeddings. We also consider a class of strong parapolar spaces, which we call near-polar spaces, first introduced by Shult [36]. In Section 3 we state a sufficient condition for an embedding of a near-polar space to be polarized (Theorem 3.1). We also prove a few lemmas on generalized quadrangles, automorphisms of projective spaces and groups generated by root groups. In Section 4, with the help of those lemmas, we will show that an $\operatorname{Aut}(\Gamma)_{0}$-homogeneous embedding of a classical dual polar space $\Gamma$ defined over a commutative division ring satisfies the sufficient condition of Theorem 3.1, thus proving Theorem 1.1. Theorem 1.2 is proved in the last part of Section 4.

\section{Definitions and basics}

\subsection{Near-polar spaces and dual polar spaces}

Throughout this paper, a point-line geometry is a pair $\Gamma=(\mathcal{P}, \mathcal{L})$ where $\mathcal{P}$ (the pointset of $\Gamma$ ) is a nonempty set, $\mathcal{L}$ (the set of lines of $\Gamma$ ) is a collection of subsets of $\mathcal{P}$, every line $l \in \mathcal{L}$ has size $|l| \geq 2$, no two lines meet in more than one point and the collinearity relation defines a connected graph on $\mathcal{P}$, called the collinearity graph of $\Gamma$. We say that a line $l$ of $\Gamma$ is thick if $|l|>2$.

The distance $d(x, y)$ between two points $x, y \in \mathcal{P}$ is the distance between them in the collinearity graph of $\Gamma$. A path of $\Gamma$ is a path of the collinearity graph of $\Gamma$. The diameter $\operatorname{diam}(\Gamma)$ of $\Gamma$ is the diameter of the collinearity graph of $\Gamma$.

A subset $S \subseteq \mathcal{P}$ is a subspace of $\Gamma$ if it contains every line $l \in \mathcal{L}$ for which $|l \cap S|>1$. A subspace $S$ is said to be proper if $S \neq \mathcal{P}$. A subspace is convex if it contains every point on a shortest path joining any two of its points. Recall that the intersection of convex subspaces is again convex. For a subset $X \subseteq \mathcal{P}$, the subspace $\langle X\rangle_{\Gamma}$ of $\Gamma$ spanned by $X$ (also generated by $X$ ) is the smallest subspace of $\Gamma$ containing $X$, namely the intersection of all subspaces of $\Gamma$ that contain $X$. The convex closure $[X]_{\Gamma}$ of $X$ is the smallest convex subspace of $\Gamma$ containing $X$. A hyperplane of $\Gamma$ is a proper subspace $H$ of $\Gamma$ such that $H \cap l \neq \emptyset$ for every line $l \in \mathcal{L}$. The following observation will be freely used throughout this paper: a hyperplane $H$ of $\Gamma$ is a maximal proper subspace of $\Gamma$ if and only if the collinearity graph of $\Gamma$ induces a connected graph on $\mathcal{P} \backslash H$.

Given a point $x \in \mathcal{P}$ and an integer $k \geq 0$, we set $\Gamma_{k}(x)=\{y \in \mathcal{P} \mid d(x, y)=k\}$ and $\Gamma_{k}^{*}(x)=\{y \in \mathcal{P} \mid d(x, y) \leq k\}$. Note that $\Gamma_{0}(x)=\{x\}$. Moreover, if $n=\operatorname{diam}(\Gamma)$ then $\Gamma_{n}^{*}(x)=\mathcal{P}$ for every point $x$. Also, $\Gamma_{m}^{*}(x)=\mathcal{P}$ and $\Gamma_{m}(x)=\emptyset$ for every $m>n$. 
In this paper we are interested in the following class of geometries, introduced by Shult [36, section 6]. Shult does not give these geometries a special name (he refers to them by the symbol $\mathcal{E}_{n}$ ), but we shall call them near-polar spaces. We say that a point-line geometry $\Gamma=(\mathcal{P}, \mathcal{L})$ is a near-polar space of diameter 1 if it is a thick line, namely $|\mathcal{L}|=1$ and $|\mathcal{P}|>2$. Let $n$ be an integer greater than 1 . We say that $\Gamma$ is a near-polar space of diameter $n$ if it satisfies all of the following:

(NP1) $\operatorname{diam}(\Gamma)=n$.

(NP2) $\Gamma_{1}(y) \cap \Gamma_{k+1}(x) \neq \emptyset$ for every nonnegative integer $k<n$ and any two points $x, y \in \mathcal{P}$ with $d(x, y)=k$.

(NP3) $\Gamma_{n-1}^{*}(x)$ is a hyperplane of $\Gamma$ for every $x \in \mathcal{P}$.

(NP4) If $x, y \in \mathcal{P}$ have distance $d(x, y)=n-1$, then the convex closure $[x, y]_{\Gamma}$ of $\{x, y\}$ carries the structure of a near-polar space of diameter $n-1$.

Near-polar spaces are strong parapolar spaces in the sense of Cooperstein and Shult [15]. A near-polar space of diameter 1 is a thick line. Hence by (NP2), (NP4) and an easy inductive argument we obtain that in a near-polar space all lines are thick. It is readily seen that the near-polar spaces of diameter 2 are just the non-degenerate polar spaces where all lines are thick. The near-polar spaces of diameter $n$ include dual polar spaces of rank $n$, projective grassmannians of type $A_{2 n-1, n}$ and half spin geometries $D_{n, n}$ with $n$ even (Shult [36]). All dense near polygons of diameter $n$ are near-polar spaces of diameter $n$ (De Bruyn [18, 23]). More examples of near-polar spaces (of diameter 3 ) are the geometries of type $E_{7,1}$ as described in Shult [36].

Proposition 2.1 All the following hold in a near-polar space $\Gamma$ of diameter $n \geq 2$.

(1) Every hyperplane of $\Gamma$ is a maximal proper subspace. In particular, for every point $x$, the set $\Gamma_{n-1}^{*}(x)$ is a maximal proper subspace of $\Gamma$.

(2) If $x, y \in \mathcal{P}$ have distance $k \neq 0$, then $[x, y]_{\Gamma}$ carries the structure of a near-polar space of diameter $k$. In particular, if $d(x, y)=2$ then $[x, y]_{\Gamma}$ carries the structure of a non-degenerate polar space.

(3) $\Gamma_{k}^{*}(x)$ is a subspace of $\Gamma$ for every $x \in \mathcal{P}$ and every $k=0,1, \ldots, n$.

(4) For every $k=0,1, \ldots, n$ and every point $x \in \mathcal{P}$, the set $\Gamma_{k}(x)$ spans $\Gamma_{k}^{*}(x)$. In particular, $\Gamma_{n}(x)$ spans the complete point-set $\mathcal{P}=\Gamma_{n}^{*}(x)$ of $\Gamma$.

Proof See Shult [36, Lemma 6.1] for (1).

We shall prove (2). If $k<n$, then Claim (2) immediately follows from (NP2) and repeated applications of (NP4). Suppose $k=n$. If $z \in[x, y]_{\Gamma} \cap \Gamma_{n}(x)$, then every line through $z$ meets $\Gamma_{n-1}(x)$ by (NP3) and hence is contained in $[x, y]_{\Gamma}$. Since the collinearity graph of $\Gamma$ induces a connected graph on $\Gamma_{n}(x)$ (see (1)), we then have that $\Gamma_{n}(x) \subseteq[x, y]_{\Gamma}$. By repeated application of (NP2), every point of $\Gamma$ is contained on a shortest path between $x$ and a point of $\Gamma_{n}(x)$. Hence, $[x, y]_{\Gamma}$ coincides with the whole point-set of $\Gamma$. So, Claim (2) is also valid in the case $k=n$.

We shall now prove (3). Let $y, z$ be collinear points of $\Gamma_{k}^{*}(x)$ and $l$ a line through them. We need to prove that $l \subseteq \Gamma_{k}^{*}(x)$. If one of $y$ or $z$ has distance less than $k$ from $x$ then there is nothing to prove. Suppose that $d(x, y)=d(x, z)=k$. If $l$ is not contained in $\Gamma_{k}^{*}(x)$ then $l$ contains a point $u \in \Gamma_{k+1}(x)$. The subspace $\Xi:=[x, u]_{\Gamma}$ contains $l$. By (2), $\Xi$ is a near-polar space of diameter $k+1$. Clearly, $y, z \in \Xi_{k}^{*}(x)$. 
By (NP3) on $\Xi$, we have $l \subseteq \Xi_{k}^{*}(x)$, contrary to the assumption that $d(x, u)=k+1$. Therefore $l \subseteq \Gamma_{k}^{*}(x)$.

We now prove (4). Let $y \in \Gamma_{h}(x)$ for $h<k$. By (NP2), there is a line $l$ on $y$ containing a point $z \in \Gamma_{h+1}(x)$. By (3), $\Gamma_{h}^{*}(x)$ is a subspace. Hence $l \cap \Gamma_{h}^{*}(x)=\{y\}$. However, lines are thick. So $l$ contains at least two points $z, z^{\prime} \in \Gamma_{h+1}(x)$. Hence $y \in\left\langle z, z^{\prime}\right\rangle_{\Gamma} \subseteq\left\langle\Gamma_{h+1}(x)\right\rangle_{\Gamma}$. Therefore $\Gamma_{h}(x) \subseteq\left\langle\Gamma_{h+1}(x)\right\rangle_{\Gamma}$ for every $h<k$. It follows that $\Gamma_{k}^{*}(x)=\left\langle\Gamma_{k}(x)\right\rangle_{\Gamma}$.

Another easy consequence of the near-polar space axioms that we will use in the proofs of Proposition 3.2 and Lemma 4.3 is the following.

Lemma 2.2 Let $x_{1}, x_{2}, y_{0}$ three distinct points on a line l of a near polar space $\Gamma$ of diameter $n \geq 2$. Then there exist points $y_{1}, y_{2}, \ldots, y_{n-1}$ such that, for each $i$ with $0 \leq i \leq n-1$, we have $d\left(x_{1}, y_{i}\right)=d\left(x_{2}, y_{i}\right)=i+1$ and $d\left(y_{0}, y_{i}\right)=i$.

Proof The points $y_{i}, i \in\{1, \ldots, n-1\}$, can be constructed in a recursive way as follows. First note that $y_{0} \in \Gamma_{1}\left(x_{1}\right) \cap \Gamma_{1}\left(x_{2}\right) \cap \Gamma_{0}\left(y_{0}\right)$. Now suppose that for a certain $i \in\{1, \ldots, n-1\}$ we have $y_{i-1} \in \Gamma_{i}\left(x_{1}\right) \cap \Gamma_{i}\left(x_{2}\right) \cap \Gamma_{i-1}\left(y_{0}\right)$. Then by (NP2) there exists a point $y_{i} \in \Gamma_{i+1}\left(x_{1}\right) \cap \Gamma_{1}\left(y_{i-1}\right)$. Combining this with $d\left(x_{1}, y_{0}\right)=$ 1 and $d\left(y_{0}, y_{i-1}\right)=i-1$, we find $y_{i} \in \Gamma_{i}\left(y_{0}\right)$ by the triangle inequality. Now $y_{0}, x_{2} \in\left[x_{1}, y_{i}\right]_{\Gamma}$ and so by Proposition 2.1(2) and (NP3), $y_{i} \in \Gamma_{i}\left(y_{0}\right) \cap \Gamma_{i+1}\left(x_{1}\right) \cap$ $\Gamma_{i+1}\left(x_{2}\right)$.

We now turn to dual polar spaces. We recall that, given a non-degenerate polar space $\Pi$ of finite rank $n \geq 1$, the dual polar space associated to $\Pi$ is the point-line geometry $\Pi^{*}=(\mathcal{P}, \mathcal{L})$ where $\mathcal{P}$ is the collection of maximal singular subspaces of $\Pi$ and the lines $l \in \mathcal{L}$ bijectively correspond to the co-maximal singular subspaces of $\Pi$. Explicitly, for every co-maximal singular subspace $S$ of $\Pi$, the set of all maximal singular subspaces of $\Pi$ containing $S$ is a line of $\Pi^{*}$, and all lines of $\Pi^{*}$ are obtained in this way. The rank $n$ of $\Pi$ is also called the rank of $\Pi^{*}$.

We finish this section by stating some more terminology and notation. Let $\Gamma$ be a near-polar space of diameter $n \geq 2$. The elements of $\mathcal{C}_{n-1}(\Gamma)$ and $\mathcal{C}_{n-2}(\Gamma)$ are called maxes and co-maxes respectively, and the elements of $\mathcal{C}_{2}(\Gamma)$ are called symplecta. As we have remarked above, symplecta are polar spaces. For $x \in \mathcal{P}$ and $k=0,1, \ldots, n$ we put $\mathcal{C}_{k}(x):=\left\{X \mid x \in X \in \mathcal{C}_{k}(\Gamma)\right\}$. So, $\mathcal{C}_{0}(x)=\{\{x\}\}$ and $\mathcal{C}_{1}(x)$ is the set of lines of $\Gamma$ through $x$. We set $\operatorname{Res}_{\Gamma}(x):=\bigcup_{k=1}^{n-1} \mathcal{C}_{k}(x)$ and we regard it as a poset, with inclusion as the partial ordering. We call $\operatorname{Res}_{\Gamma}(x)$ the residue of $x$. We also put $H_{x}:=$ $\Gamma_{n-1}^{*}(x)$ and we call $H_{x}$ the singular hyperplane of $\Gamma$ with $x$ as its deep point.

Let $\Gamma$ be a dual polar space, say $\Gamma=\Pi^{*}$ for a polar space $\Pi$ of rank $n \geq 2$. Then every symplecton of $\Gamma$ carries the structure of a generalized quadrangle. Following a well established custom, we call the symplecta of $\Gamma$ quads, setting aside the word 'symplecton' in this context. The maxes of $\Gamma$ correspond to the points of $\Pi$. More generally, if $n=\operatorname{diam}(\Gamma)=\operatorname{rank}(\Pi)$, then the members of $\mathcal{C}_{k}(\Gamma)$ correspond to the singular subspaces of $\Pi$ of rank $n-k$. (We recall that the rank of a singular subspace $S$ of $\Pi$ is $\operatorname{dim}(S)+1$, where $\operatorname{dim}(S)$ is the projective dimension of $S$ ). If $x \in \mathcal{P}$ then $\operatorname{Res}_{\Gamma}(x)$ is a projective geometry of dimension $n-1$. 
A dual polar space $\Gamma=\Pi^{*}$ is thick if $\Pi$ is thick, namely all lines of $\Gamma$ are thick (so, $\Gamma$ is a near-polar space) and every co-max is contained in at least three maxes. We say that $\Gamma$ is classical if $\Pi$ is classical, namely $\Pi$ is embeddable in the sense of the next subsection. If $\Gamma$ is classical and embeddable then $\Gamma$ is thick.

\subsection{Embeddings}

A full projective embedding (or embedding for short) of a point-line geometry $\Gamma=$ $(\mathcal{P}, \mathcal{L})$ into a Desarguesian projective space $\Sigma$ (embedding of $\Gamma$ in $\Sigma$, for short) is an injective mapping $e$ from the point-set $\mathcal{P}$ of $\Gamma$ to the point-set of $\Sigma$ satisfying the following:

(E1) the image $e(\mathcal{P})$ of $\mathcal{P}$ by $e$ spans $\Sigma$;

(E2) every line of $\Gamma$ is mapped by $e$ onto a line of $\Sigma$.

The numbers $\operatorname{dim}(\Sigma)$ and $\operatorname{dim}(\Sigma)+1$ are respectively called the projective dimension and the vector dimension of the embedding $e$. The underlying division ring $\mathbb{F}$ of $\Sigma$ is called the underlying division ring of $e$ and we say that $e$ is defined over $\mathbb{F}$, also that $e$ is an $\mathbb{F}$-embedding for short. If $\Gamma$ admits an embedding (an $\mathbb{F}$-embedding) then we say that $\Gamma$ is embeddable ( $\mathbb{F}$-embeddable). Obviously, if $\Gamma$ is embeddable then all lines of $\Gamma$ are thick. More explicitly, if $\Gamma$ is $\mathbb{F}$-embeddable then every line of $\Gamma$ has $|\mathbb{F}|+1$ points.

Let $\Gamma$ be embeddable. If, up to isomorphism, the underlying division ring $\mathbb{F}$ of an embedding $e$ of $\Gamma$ does not depend on the particular choice of the embedding $e$ then we say that $\Gamma$ is defined over $\mathbb{F}$. We also say that $\mathbb{F}$ is the underlying division ring of $\Gamma$. For instance, this holds when the lines of $\Gamma$ have a finite number of points. It also holds when $\Gamma$ is an embeddable non-degenerate polar space of rank $n \geq 3$ and when $\Gamma$ is an embeddable non-degenerate thick generalized quadrangle. In the latter case, the existence of the underlying division ring is a by-product of the existence of the absolutely universal embedding (Buekenhout and Lefèvre [8], Dienst [27] and Tits [38, Theorem 8.6]; see below for the definition of the absolutely universal embedding). As a consequence of the above, every embeddable near-polar space of diameter $n \geq 2$ with thick symplecta admits an underlying division ring. In particular, every embeddable thick dual polar space is defined over some division ring.

\subsubsection{Isomorphisms and quotients of embeddings}

Two embeddings $e_{1}: \Gamma \rightarrow \Sigma_{1}$ and $e_{2}: \Gamma \rightarrow \Sigma_{2}$ of a point-line geometry $\Gamma$ are said to be isomorphic (and we write $e_{1} \cong e_{2}$ ) if there exists an isomorphism $f: \Sigma_{1} \rightarrow \Sigma_{2}$ such that $e_{2}=f \circ e_{1}$.

Let $e: \Gamma \rightarrow \Sigma$ be an embedding of $\Gamma$ and $U$ a subspace of $\Sigma$ satisfying the following:

(Q1) $\langle U, e(x)\rangle \neq U$ for every point $x \in \mathcal{P}$;

(Q2) $\langle U, e(x)\rangle \neq\langle U, e(y)\rangle$ for any two distinct points $x, y \in \mathcal{P}$.

Then there exists an embedding $e / U$ of $\Gamma$ into the quotient space $\Sigma / U$, mapping each point $x$ of $\Gamma$ to $\langle U, e(x)\rangle$. We call $e / U$ the quotient of $e$ by $U$. If $e_{1}: \Gamma \rightarrow \Sigma_{1}$ and $e_{2}: \Gamma \rightarrow \Sigma_{2}$ are two embeddings, we say that $e_{1} \geq e_{2}$ if there exists a subspace $U$ in $\Sigma_{1}$ satisfying (Q1), (Q2) and $e_{1} / U \cong e_{2}$. 
Proposition 2.3 (See [32, Proposition 10]) Let $e$ and $\Gamma$ be as above. Then no two distinct quotients of e are isomorphic.

\subsubsection{Hulls, relatively and absolutely universal embeddings}

Every embedding $e: \Gamma \rightarrow \Sigma$ admits a hull $\tilde{e}: \Gamma \rightarrow \widetilde{\Sigma}$ uniquely determined up to isomorphism by the following property (Ronan [34]): $\tilde{e} \geq e$ and, if $e^{\prime}: \Gamma \rightarrow \Sigma^{\prime}$ is an embedding such that $e^{\prime} \geq e$, then $\tilde{e} \geq e^{\prime}$. The hull $\tilde{e}$ of $e$ is also said to be relatively universal with respect to $e$.

An embedding $\tilde{e}: \Gamma \rightarrow \widetilde{\Sigma}$ is said to be absolutely universal (also absolute, for short) if it is the hull of every embedding of $\Gamma$. Clearly, absolute embeddings, when they exist, are uniquely determined up to isomorphism. It is also clear that $\Gamma$ admits the absolute embedding only if it admits an underlying division ring.

It is well known that every embeddable non-degenerate polar space of rank at least 2 which is not a grid admits the absolutely universal embedding (Buekenhout and Lefèvre [8], Dienst [27] and Tits [38, 8.6]). Kasikova and Shult [29, 4.6] have proved that all thick embeddable dual polar spaces admit the absolutely universal embedding.

\subsubsection{Homogeneity}

Given an embedding $e: \Gamma \rightarrow \Sigma$ we say that an automorphism $g$ of $\Gamma$ lifts to $\Sigma$ through $e$ if there exists an automorphism $e(g)$ of $\Sigma$ such that $e(g) e=e g$. Clearly $e(g)$, if it exists, is uniquely determined by $g$. We call it the lifting of $g$ to $\Sigma$. Let $G$ be a subgroup of $\operatorname{Aut}(\Gamma)$. If all elements of $G$ lift to $\Sigma$ through $e$ then we say that $G$ lifts to $\Sigma$, we put $e(G)=\{e(g)\}_{g \in G}$ and we call $e(G)$ the lifting of $G$ to $\Sigma$ (also, the lifting of $G$ to $\operatorname{Aut}(\Sigma))$. Notice that $e(G)$ is indeed a group. If $G$ lifts to $\Sigma$ through $e$ then we also say that $e$ is $G$-homogeneous. If $e$ is $G$-homogeneous, then a subspace $U$ of $\Sigma$ is said to be stabilized by $G$ (also, to be $G$-invariant), if it is stabilized by $e(G)$. Clearly, if a subspace $U$ of $\Sigma$ defines a quotient of $e$ and it is $G$-invariant, then $e / U$ is $G$-homogeneous.

Proposition 2.4 (See [32, Lemma 12]) Assume that e is G-homogeneous. Let $U$ be a subspace of $\Sigma$ defining a quotient of e and suppose that e/ $U$ is $G$-homogeneous. Then $U$ is $G$-invariant.

If all automorphisms of $\Gamma$ lift to $\Sigma$ through $e$ then we say that $e$ is fully homogeneous. Suppose that $\Gamma$ admits the absolutely universal embedding $\tilde{e}: \Gamma \rightarrow \widetilde{\Sigma}=$ $\operatorname{PG}(\widetilde{V})$. Then $\tilde{e}$ is fully homogeneous. In this case, if $G$ is a subgroup of $\operatorname{Aut}(\Gamma)$, we put $G_{\text {lin }}:=\tilde{e}^{-1}(\tilde{e}(G) \cap \operatorname{PGL}(\widetilde{V}))$ and we call $G_{\text {lin }}$ the linear part of $G$. It is not difficult to see that $G_{\text {lin }}$ is a normal subgroup of $G$. We say that $G$ is linear if $G=G_{\text {lin }}$.

\subsubsection{Polarized embeddings}

Let $\Gamma=(\mathcal{P}, \mathcal{L})$ be a near-polar space of diameter $\operatorname{diam}(\Gamma)=n>1$ and let $e: \Gamma \rightarrow \Sigma$ be an embedding of $\Gamma$. It is known that all hyperplanes of a near-polar space $\Gamma$ are 
maximal subspaces of $\Gamma$ (Shult [36, Lemma 6.1]). Therefore, if $H$ is a hyperplane of $\Gamma$, then $S_{H}=\langle e(H)\rangle$ is either a hyperplane of $\Sigma$ or the whole point-set of $\Sigma$. We say that $e$ is polarized if $S_{H}$ is a hyperplane of $\Sigma$ for every singular hyperplane $H$ of $\Gamma$ (recall that $H$ is singular if $H=H_{x}=\Gamma_{n-1}^{*}(x)$ for a point $x \in \mathcal{P}$ ).

Suppose that $e$ is polarized and let $R_{e}$ be the intersection of all hyperplanes $\left\langle e\left(H_{x}\right)\right\rangle$ of $\Sigma$ for $x \in \mathcal{P}$. Following [3], we call $R_{e}$ the nucleus of $e$.

Proposition 2.5 De Bruyn [23] Let e be polarized. Then $R_{e}$ defines a quotient of $e$.

Suppose that $\Gamma$ admits the absolutely universal embedding $\tilde{e}: \Gamma \rightarrow \widetilde{\Sigma}$ and $\tilde{e}$ is polarized. So, $R_{\tilde{e}}$ defines a quotient of $\tilde{e}$. Then $\bar{e}:=\tilde{e} / R_{\tilde{e}}$ is a polarized embedding of $\Gamma$, called the minimal polarized embedding of $\Gamma$. If $e=\tilde{e} / U$ is another embedding of $\Gamma$, then $e$ is polarized if and only if $U \subseteq R_{\tilde{e}}$, namely $e \geq \bar{e}$. Suppose this is the case. Then $R_{e}=R_{\tilde{e}} / U, R_{e}$ defines a quotient of $e$ and $e / R_{e} \cong \bar{e}$. Note also that $\bar{e}$ is fully homogeneous. Indeed, $R_{\tilde{e}}$ is stabilized by the lifting of $\operatorname{Aut}(\Gamma)$ to $\widetilde{\Sigma}$.

So far for a general near-polar space. We shall now turn to polar spaces and dual polar spaces. The following proposition is well known. It can be obtained from the theory of Veldkamp spaces of polar spaces (see Buekenhout and Cohen [7, chapters 9-12]). Another argument is given in [28, Proposition 5.4].

Proposition 2.6 All embeddings of non-degenerate polar spaces are polarized.

Let $\Gamma$ be a non-degenerate thick polar space and let $e: \Gamma \rightarrow \Sigma$ be an embedding of $\Gamma$. The embedding $e$ is polarized by Proposition 2.6. By Proposition 2.5, the nucleus $R_{e}$ of $e$ defines a quotient of $e$. As $\Gamma$ admits the absolute embedding, it also admits the minimal polarized embedding $\bar{e}$ and we have $e / R_{e} \cong \bar{e}$.

Let now $\Gamma$ be a thick dual polar space and $\tilde{e}: \Gamma \rightarrow \widetilde{\Sigma}$ be its absolute embedding. The embedding $\tilde{e}$ is polarized by Cardinali, De Bruyn and Pasini [11, Corollary 1.8]). By Proposition $2.5, R_{\tilde{e}}$ defines a quotient of $\Gamma$. Hence $\Gamma$ admits the minimal polarized embedding.

\subsection{Tangent spaces}

Let $e: \Gamma \rightarrow \operatorname{PG}(V)$ be an embedding of a near-polar space $\Gamma=(\mathcal{P}, \mathcal{L})$, with $\operatorname{diam}(\Gamma)=n>1$. Following Cardinali and De Bruyn [9], for every point $x \in \mathcal{P}$ we define $T_{-1}(x):=0$ (vector notation) and, for every $i=0,1, \ldots, n$ we put $T_{i}(x):=$ $\left\langle e\left(\Gamma_{i}^{*}(x)\right)\right\rangle$ (regarded as a subspace of $V$ ). In particular, $T_{n}(x)=\left\langle e\left(\Gamma_{n}^{*}(x)\right)\right\rangle=V$. The subspace $T_{i}(x)$ is called the tangent space of $e$ of degree $i$ at $e(x)$. Clearly, $T_{i-1}(x) \subseteq T_{i}(x)$ for every $i=0,1, \ldots, n$. We put $M_{i}(x):=T_{i}(x) / T_{i-1}(x)$ and we call $M_{i}(x)$ the reduced tangent space of $e$ of degree $i$ at $e(x)$. In particular, $M_{0}(x)=e(x)$. Note that the set $\left\{e(y)+T_{i-1}(x)\right\}_{y \in \Gamma_{i}(x)}$ spans $M_{i}(x)$. Note also that $e$ is polarized if and only if $M_{n}(x) \neq 0$ for every point $x$. For every $C \in \mathcal{C}_{i}(x)$ put

$$
p_{i}(C):=\frac{\langle e(C)\rangle+T_{i-1}(x)}{T_{i-1}(x)} .
$$

The next proposition rephrases a result by Cardinali and De Bruyn [9]. 
Proposition 2.7 Suppose $M_{i}(x) \neq 0$ for a given point $x \in \mathcal{P}$ and an index $i \in\{0,1, \ldots, n\}$. Then $p_{i}(C)$ has vector dimension $\operatorname{dim}\left(p_{i}(C)\right) \leq 1$ for every $C \in \mathcal{C}_{i}(x)$. Moreover, $\left\{p_{i}(C) \mid C \in \mathcal{C}_{i}(x), p_{i}(C) \neq 0\right\}$ is a spanning set of points of $\mathrm{PG}\left(M_{i}(x)\right)$.

Proof We may assume that $i \geq 1$. Let $C \in \mathcal{C}_{i}(x)$. Then $C$ carries the structure of a near-polar space. By (NP3), every line of $C$ meets $\Gamma_{i-1}^{*}(x)$. Moreover, $H_{x}^{C}:=$ $C \cap \Gamma_{i-1}^{*}(x)$ is the singular hyperplane of $C$ with $x$ as the deep point. By (1) of Proposition 2.1, $H_{x}^{C}$ is a maximal proper subspace of $C$. Hence the collinearity relation of $C$ induces a connected graph on $C \backslash H_{x}^{C}$. Therefore either $e(C) \subseteq T_{i-1}(x)$ or $T_{i-1}(x)$ is a hyperplane of $\langle e(C)\rangle+T_{i-1}(x)$, namely $p_{i}(C)$ is either the null subspace of $M_{i}(x)$ or a point of $\mathrm{PG}\left(M_{i}(x)\right)$.

Since $\bigcup_{C \in \mathcal{C}_{i}(x)} e(C)$ spans $T_{i}(x),\left\{p_{i}(C) \mid C \in \mathcal{C}_{i}(x), p_{i}(C) \neq 0\right\}$ spans $\operatorname{PG}\left(M_{i}(x)\right)$.

Assume that $\Gamma$ is a dual polar space. For $i \in\{0,1, \ldots, n\}, \mathcal{C}_{i}(x)$ can be regarded as the set of $(i-1)$-dimensional subspaces of the $(n-1)$-dimensional projective geometry $\operatorname{Res}_{\Gamma}(x)$. In particular, the elements of $\mathcal{C}_{1}(x)$ (lines of $\Gamma$ through $x$ ) are the points of $\operatorname{Res}_{\Gamma}(x)$ and the elements of $\mathcal{C}_{n-1}(x)$ (maxes of $\Gamma$ on $x$ ) are the hyperplanes of $\operatorname{Res}_{\Gamma}(x)$.

We denote by $\mathcal{G}_{i}(x), 1 \leq i \leq n-1$, the $i$-grassmannian of $\operatorname{Res}_{\Gamma}(x)$. So, $\mathcal{C}_{i}(x)$ is the set of points of $\mathcal{G}_{i}(x)$. The lines of $\mathcal{G}_{i}(x)$ bijectively correspond to the pairs $\{A, B\}$ with $A \in \mathcal{C}_{i-1}(x), B \in \mathcal{C}_{i+1}(x)$ and $A \subset B$, where $\left\{C \in \mathcal{C}_{i}(x) \mid A \subset C \subset B\right\}$ is the line of $\mathcal{G}_{i}(x)$ corresponding to $\{A, B\}$.

Proposition 2.8 (Cardinali and De Bruyn [9]) Assume that $\Gamma$ is a dual polar space and $e$ is polarized. Let $i \in\{1,2, \ldots, n-1\}$. Then $\operatorname{dim}\left(M_{i}(x)\right)>1, p_{i}(C) \neq 0$ for every $C \in \mathcal{C}_{i}(x)$ and $p_{i}$ is an injective mapping from the point-set $\mathcal{C}_{i}(x)$ of $\mathcal{G}_{i}(x)$ to the set of points of $\mathrm{PG}\left(M_{i}(x)\right)$.

With $\Gamma$ and $e$ as in Proposition 2.8, the image by $p_{i}$ of a line of $\mathcal{G}_{i}(x)$ is a curve of $\operatorname{PG}\left(M_{i}(x)\right)$, whose type depends on the type of $\Gamma$ and features of $e$. We are not going to discuss this matter here. We refer to Cardinali and De Bruyn [9] for more details.

\section{Preliminary results}

\subsection{A theorem on embeddings of near-polar spaces}

Let $\Gamma=(\mathcal{P}, \mathcal{L})$ be a near-polar space of diameter $n>1$ and let $e: \Gamma \rightarrow \Sigma$ be an embedding of $\Gamma$. Let $G$ be a subgroup of $\operatorname{Aut}(\Gamma)$ which lifts through $e$ to a subgroup $e(G)$ of $\operatorname{Aut}(\Sigma)$. For a point $x \in \mathcal{P}$, we denote by $G_{x}$ the stabilizer of $x$ in $G$. Clearly, if $X$ is a subgroup of $G_{x}$, then $e(X)$ stabilizes the tangent space $T_{i}(x)$ for every $i=$ $-1,0, \ldots, n$. Accordingly, with $M_{i}(x)=T_{i}(x) / T_{i-1}(x)$ as in the previous section, $e(X)$ acts on $\operatorname{PG}\left(M_{i}(x)\right)$ for every $i=0,1, \ldots, n$. 
Theorem 3.1 Assume the following:

$(\dagger)$ For every point $x \in \mathcal{P}$, the stabilizer $G_{x}$ of $x$ in $G$ admits a subgroup $K_{x}$ such that $K_{x}$ acts transitively on $\Gamma_{n}(x)$ and $e\left(K_{x}\right)$ acts trivially on $\operatorname{PG}\left(M_{i}(x)\right)$ for every $i=1,2, \ldots, n-1$.

Then e is polarized.

Proof By way of contradiction, suppose that $e$ is not polarized. Then there exists a point $x \in \mathcal{P}$ such that $T_{n-1}(x)=\Sigma$. (Here and throughout the rest of this proof we regard tangent spaces as subspaces of $\Sigma$.) However, $T_{0}(x)=e(x) \neq \Sigma$. So, $T_{i}(x) \neq \Sigma$ for some $i$. Let $k$ be the largest index $i(<n-1)$ such that $T_{i}(x) \neq \Sigma$. Then $T_{k+1}(x)=\Sigma$, whence $e(y) \in T_{k+1}(x)$ for every $y \in \Gamma_{n}(x)$. The group $e\left(K_{x}\right)$ acts trivially on $\mathrm{PG}\left(M_{k+1}(x)\right)$, by the second part of $(\dagger)$. Therefore, denoted by $K_{x}(e(y))$ the $e\left(K_{x}\right)$-orbit of $e(y)$, we have $\left\langle K_{x}(e(y)) \cup T_{k}(x)\right\rangle=\left\langle e(y), T_{k}(x)\right\rangle$. On the other hand, $K_{x}$ acts transitively on $\Gamma_{n}(x)$. Hence $\left\langle e(y), T_{k}(x)\right\rangle=\left\langle e(z), T_{k}(x)\right\rangle$ for any two points $y, z \in \Gamma_{n}(x)$. Moreover, $\Gamma_{n}(x)$ spans $\Gamma$ (claim (4) of Proposition 2.1). It follows that $T_{k}(x)$ is a hyperplane of $\Sigma$. Also, $e(y) \notin T_{k}(x)$ for every point $y \in \Gamma_{n}(x)$.

Let now $z$ be a point of $\Gamma_{n-1}(x)$ and $l$ a line of $\Gamma$ joining $z$ with a point $y$ of $\Gamma_{n}(x)$. Such a line exists by (NP2). Note that all points of $l$ but $z$ belong to $\Gamma_{n}(x)$, by (NP3). As $T_{k}(x)$ is a hyperplane of $\Sigma$, the line $e(l)$ of $\Sigma$ meets $T_{k}(x)$ in a point $p$. We have $p=e\left(z^{\prime}\right)$ for a point $z^{\prime} \in l$ because $e$ maps $l$ onto $e(l)$. However, if $z^{\prime} \neq z$ then $z^{\prime} \in \Gamma_{n}(x)$. Hence $e\left(z^{\prime}\right) \notin T_{k}(x)$. It follows that $z^{\prime}=z$. So, $e(z) \in T_{k}(x)$ for every $z \in \Gamma_{n-1}(x)$. Moreover, $\Gamma_{n-1}(x)$ spans $\Gamma_{n-1}^{*}(x)$ by (4) of Proposition 2.1. Therefore $T_{n-1}(x) \subseteq T_{k}(x)$. Since $T_{n-1}(x)=\Sigma$, also $T_{k}(x)=\Sigma$. We have reached a contradiction.

We now state an easy consequence of Property $(\dagger)$. It is irrelevant for the proof of Theorem 1.1, but the proof contains an idea that will be recycled in the last lemma of this paper (in Section 4).

Proposition 3.2 Suppose that $G$ satisfies property $(\dagger)$ of Theorem 3.1. Then $G$ acts transitively on $\mathcal{P}$.

Proof This follows from the connectedness of $\Gamma$ and the fact that given any two distinct collinear points $x_{1}$ and $x_{2}$, there is a point $y$ with $x_{1}, x_{2} \in \Gamma_{n}(y)$ by Lemma 2.2.

\subsection{A lemma on generalized quadrangles}

Let $\Gamma=(\mathcal{P}, \mathcal{L})$ be a thick embeddable generalized quadrangle and let $e: \Gamma \rightarrow \operatorname{PG}(V)$ be an embedding of $\Gamma$. Let $\mathbb{F}$ be the underlying division ring of $\Gamma$. As in Section 2, for a point $x \in \mathcal{P}$ we put $M_{1}(x)=T_{1}(x) / e(x)=T_{1}(x) / T_{0}(x)$ and we denote by $\mathcal{C}_{1}(x)$ the set of lines of $\Gamma$ on $x$. So, the elements of $\mathcal{C}_{1}(x)$ can be regarded as points of $\operatorname{PG}\left(M_{1}(x)\right)$.

Lemma 3.3 Suppose $\mathbb{F} \neq \mathbb{F}_{2}$. Given a point $x \in \mathcal{P}$, let $l_{1}$ and $l_{2}$ be two distinct members of $\mathcal{C}_{1}(x)$ and let $L$ be the line of $\mathrm{PG}\left(M_{1}(x)\right)$ through them. Then one of the following holds: 
(1) $\left|L \cap \mathcal{C}_{1}(x)\right|>2$;

(2) There is a line $L^{\prime}$ of $\operatorname{PG}\left(M_{1}(x)\right)$ such that $\left|L^{\prime} \cap \mathcal{C}_{1}(x)\right| \geq 2$ and $L^{\prime}$ meets $L$ in a point $p \notin \mathcal{C}_{1}(x)$.

Proof Let $\tilde{e}: \Gamma \rightarrow \operatorname{PG}(\tilde{V})$ be the absolutely universal embedding of $\Gamma$. So, $e \cong \tilde{e} / U$ for a subspace $U$ of $\widetilde{V}$. If $l_{1}$ and $l_{2}$ are distinct members of $\mathcal{C}_{1}(x)$, then the 2dimensional subspace of $\widetilde{V}$ spanned by $\tilde{e}\left(l_{1}\right) \cup \tilde{e}\left(l_{2}\right)$ meets $U$ trivially. It follows that, if the statement of the lemma holds for $\tilde{e}$, then it also holds for $e$. So, we may safely assume that $e=\tilde{e}$, namely $e$ is absolutely universal.

As $e$ is absolute, $\Gamma$ arises from a non-degenerate reflexive sesquilinear form $f$ of $V$ or a non-singular pseudo-quadratic form $q$ of $V$ (Buekenhout and Lefèvre [8], Dienst [27], Tits [38, 8.6]; see also Buekenhout and Cohen [7, chapter 11]).

Suppose that (1) is false for the given lines $l_{1}, l_{2} \in \mathcal{C}_{1}(x)$. Then, considering the 2-dimensional subspace of $\widetilde{V}$ spanned by $\tilde{e}\left(l_{1}\right) \cup \tilde{e}\left(l_{2}\right)$ we see that the sesquilinear form $f$ or the sesquilinear form associated to $q$ is in fact symmetric bilinear so that $\Gamma$ is quadratic. It follows that $\widetilde{V}$ has dimension at least 5 . In the latter case we can consider the non-degenerate sub-quadrangle induced on some suitably chosen 5-dimensional subspace of $\widetilde{V}$ containing $x$. Hence it suffices to check that (2) holds in case $\Gamma$ is quadratic and $\widetilde{V}$ has dimension 5. Now, $\mathcal{C}_{1}(x)$ is a conic of the Pappian plane $\mathrm{PG}\left(M_{1}(x)\right)$ and the claim follows.

\subsection{A lemma on projective spaces}

Given a vector space $V$ over a division ring $\mathbb{F}$, let $\Sigma=\mathrm{PG}(V)$. We do not assume $\mathbb{F}$ to be commutative and we allow $\operatorname{dim}(\Sigma)$ to be infinite. Denoted by $\mathcal{P}$ the point-set of $\Sigma$, let $\mathcal{G}=(S, \sim)$ be a graph defined on a subset $S \subseteq \mathcal{P}$ such that (S1) $S$ spans $\Sigma$ and $(\mathrm{S} 2) \mathcal{G}$ is connected.

Lemma 3.4 With $\Sigma, S$ and $\mathcal{G}$ as above, let $g \in \operatorname{Aut}(\Sigma)$ be such that:

(1) $g$ fixes all points of $S$;

(2) if $\left\{p_{1}, p_{2}\right\}$ is an edge of $\mathcal{G}$, then $g$ fixes all points of the line $\left\langle p_{1}, p_{2}\right\rangle$ of $\Sigma$.

Then $g$ fixes all points of $\Sigma$.

Proof We first quickly prove the following well-known fact.

(F) If $\operatorname{dim}(V)=n \geq 3$, then the only automorphism of $\Sigma$ fixing both a hyperplane and a line off that hyperplane point-wise is the identity.

Let $g$ be an automorphism of $\Sigma$ that fixes both a hyperplane $H$ and a line $k$ off $H$ point-wise. Now any point $p \in \Sigma$ not in $H \cup k$ lies on two distinct lines $l$ and $m$ meeting $k$ and $H$ in four distinct points. Those four points are fixed, hence so are $l$ and $m$ and their intersection point $p$.

Taking $n=3$ in (F), and using that $\mathcal{G}$ is connected by (S2), we find that $g$ also satisfies conditions (1) and (2) for the complete graph on the vertex set $S$. Thus all lines meeting $S$ in at least two points are fixed point-wise by $g$.

If $p$ is a point in the span of $S$, then it lies in a subspace $\Sigma^{\prime}$ of $\Sigma$ spanned by a finite number $n$ of points in $S$. The cases $n=1,2$ being trivial, we use (F) and induction on $n \geq 3$ to see that $g$ fixes $p$. We are done by (S1). 


\subsection{Root groups and the group $\operatorname{Aut}_{0}(\Delta)$}

In this subsection $\Pi$ is a non-degenerate thick polar space of rank $n \geq 2$ and $\Gamma$ is its dual. We assume that both $\Pi$ and $\Gamma$ are embeddable. As we have remarked earlier, both $\Pi$ and $\Gamma$ admit the absolutely universal embedding. Thus, both $\operatorname{Aut}(\Pi)_{\text {lin }}$ and Aut $(\Gamma)_{\text {lin }}$ are defined (Subsection 2.2.3). Let $\Delta=\Delta(\Pi)=\Delta(\Gamma)$ be the building (of type $C_{n}$ ) associated to $\Pi$ and $\Gamma$. The groups $\operatorname{Aut}(\Delta), \operatorname{Aut}(\Pi)$ and $\operatorname{Aut}(\Gamma)$ are distinct faithful actions of the same abstract group. To be pedantic, we should keep them distinct, but we will neglect these distinctions, thus regarding $\operatorname{Aut}(\Delta), \operatorname{Aut}(\Pi)$ and $\operatorname{Aut}(\Gamma)$ as the same group, as many people do. Accordingly, we regard $\operatorname{Aut}(\Pi)_{\text {lin }}$ and $\operatorname{Aut}(\Gamma)_{\text {lin }}$ as subgroups of $\operatorname{Aut}(\Delta)(=\operatorname{Aut}(\Pi)=\operatorname{Aut}(\Gamma))$. Note that $\operatorname{Aut}(\Pi)_{\text {lin }}$ and $\operatorname{Aut}(\Gamma)_{\text {lin }}$ might be different. This can happen when $\Pi$ and $\Gamma$ are defined over non-isomorphic division rings.

In this subsection we will freely use some terminology from the theory of buildings. Since the concepts such as chambers, apartments, residues, panels, roots and walls are well-known, we will not go into any detail, but refer the reader to Tits [38], Ronan [35], and Weiss [40] for the general theory. For a detailed description of the building of type $C_{n}$ associated to a polar space, its automorphism groups and $B N$ pairs, we refer to Taylor [37].

As we have already remarked in Section 2, the polar space $\Pi$, being embeddable, arises from a sesquilinear or pseudoquadratic form. Since it is thick, its building $\Delta=\Delta(\Pi)$ is Moufang (Tits [38, page 274], Ronan [35], Weiss [40]; also Tits and Weiss [39] for the case $n=2)$. Therefore, it makes sense to define $\operatorname{Aut}(\Delta)_{0}$ to be the subgroup of $\operatorname{Aut}(\Delta)$ generated by the root subgroups. This group is simple except in three cases (see Weiss $[40,12.20]$ ). The only exception that occurs among the cases we are considering is when $\operatorname{Aut}(\Delta)_{0}=\mathrm{Sp}_{4}\left(\mathbb{F}_{2}\right)$, but the derived group has index 2 . In all other cases under consideration, $\operatorname{Aut}(\Delta)_{0}$ is the largest normal simple subgroup of $\operatorname{Aut}(\Delta)$.

It readily follows from the definition of root subgroups that every root subgroup is contained in $\operatorname{Aut}(\Pi)_{\text {lin }} \cap \operatorname{Aut}(\Gamma)_{\text {lin }}$. Therefore,

Lemma $3.5 \operatorname{Aut}(\Delta)_{0} \leq \operatorname{Aut}(\Pi)_{\text {lin }} \cap \operatorname{Aut}(\Gamma)_{\text {lin }}$.

In other words, $\operatorname{Aut}(\Delta)_{0}$ is linear, no matter if it is regarded as a group of automorphisms of $\Pi$ or of $\Gamma$.

The group $\operatorname{Aut}(\Delta)_{0}$ acts transitively on the set of pairs $(c, \mathcal{A})$ where $\mathcal{A}$ is an apartment of $\Delta$ and $c$ is a chamber of $\mathcal{A}$ (Ronan [35, Ch. 6], Weiss [40, 11.12]). It follows that $\operatorname{Aut}(\Delta)_{0}$ admits a $B N$-pair associated with $\Delta$ (Tits [38], Ronan [35, Ch. 5], Weiss [40]). We call the above transitivity property $B N$-transitivity. In the case of a spherical building this coincides with what is called strong-transitivity in the literature. In the present paper, the most important consequence of this is that $\operatorname{Aut}(\Delta)_{0}$ is transitive on the set of ordered pairs $\left(C_{1}, C_{2}\right) \in \mathcal{C}_{d_{1}}(\Gamma) \times \mathcal{C}_{d_{2}}(\Gamma)$ (notation as in Subsection 2.1) with $C_{1} \cap C_{2} \in \mathcal{C}_{d_{3}}(\Gamma)$ and $\left[C_{1} \cup C_{2}\right]_{\Gamma} \in \mathcal{C}_{d_{4}}(\Gamma)$, for any choice of $d_{1}, d_{2}, d_{3}, d_{4}$ with $-1 \leq d_{3} \leq d_{1}, d_{2} \leq d_{4} \leq n$ and $0 \leq d_{1}, d_{2} \leq n-1$. Here, we have taken the convention that $\mathcal{C}_{-1}(\Gamma)=\{\emptyset\}$. 
Lemma 3.6 For a point $x$ of $\Gamma$, let $G_{x}$ be the stabilizer of $x$ in $G:=\operatorname{Aut}(\Delta)_{0}$ and let $K_{x}$ be the elementwise stabilizer of $\operatorname{Res}_{\Gamma}(x)$ in $G_{x}$. Then both the following hold:

(1) the group $G_{x} / K_{x}$ induced by $G_{x}$ on $\operatorname{Res}_{\Gamma}(x)$ is $B N$-transitive as a subgroup of $\operatorname{Aut}\left(\operatorname{Res}_{\Gamma}(x)\right)$;

(2) $K_{x}$ acts simply transitively on $\Gamma_{n}(x)$.

Proof By strong-transitivity we may assume that $x$ lies on the chamber $c$ of the apartment $\mathcal{A}$ such that $G=\left\langle U_{\alpha} \mid \alpha \in \Phi(\mathcal{A})\right\rangle, B=\operatorname{Stab}_{G}(c)$ and $N=\operatorname{Stab}_{G}(\mathcal{A})$. Here $\Phi(\mathcal{A})$ is the set of all roots in $\mathcal{A}$ and $U_{\alpha}$ is the root group of the root $\alpha$. Let $\Phi_{x}$ consist of those roots $\alpha$ of $\mathcal{A}$ such that $U_{\alpha}$ fixes $x$. Then partition $\Phi_{x}=\Phi_{x}^{0} \uplus \Phi_{x}^{+}$ $\left(\biguplus\right.$ denotes disjoint union). Here $\Phi_{x}^{+}$consists of those roots $\alpha$ of $\mathcal{A}$ such that every chamber of $\mathcal{A}$ on $x$ belongs to $\alpha$ and $\Phi_{x}^{0}$ denotes the set of roots of $\mathcal{A}$ such that $x$ lies on the wall $\partial$ of $\alpha$. In other words, $\Phi_{x}^{+}$consists of those roots $\alpha$ of $\mathcal{A}$ such that $U_{\alpha}$ fixes every chamber of $\mathcal{A}$ on $x$.

Define $U_{x}=\left\langle U_{\alpha} \mid \alpha \in \Phi_{x}^{+}\right\rangle$and $L_{x}=\left\langle U_{\alpha}, B \cap N \mid \alpha \in \Phi_{x}^{0}\right\rangle$. Then Levi decomposition (cf. Theorem 6.18 of [35]) means that we have a semi-direct product $G_{x}=U_{x} \rtimes L_{x}$. Interpreting the results to our setting, it is proved in loc. cit. that $U_{x}$ acts regularly on the points opposite to $x$, i.e. at distance $n$ from $x$. Moreover, $L_{x}$ induces the full group generated by root groups of the Moufang building that is $x$ itself. In particular, $L_{x}$ acts strongly transitively on the residue $\operatorname{Res}_{\Gamma}(x)$. Now (1) follows. Since by definition $U_{x}$ fixes every chamber of $\mathcal{A}$ on $x$, and is normalized by $L_{x}$ the strong-transitivity of $L_{x}$ on $\operatorname{Res}_{\Gamma}(x)$ implies that $U_{x}$ in fact fixes every chamber on $x$. Thus, $U_{x} \leq K_{x}$ and (2) follows.

\section{Proofs of Theorems 1.1 and 1.2}

\subsection{Proof of Theorem 1.1}

Let $\Gamma=(\mathcal{P}, \mathcal{L})$ be an embeddable classical dual polar space of rank $n \geq 2$ defined over a commutative division ring $\mathbb{F}$, let $e: \Gamma \rightarrow \mathrm{PG}(V)$ be a $G$-homogeneous embedding of $\Gamma$, with $G=\operatorname{Aut}(\Gamma)_{0}:=\operatorname{Aut}(\Delta)_{0}$ and $\Delta=\Delta(\Gamma)$ as in Subsection 3.4. We know by Lemma 3.5 that $G$ is linear. We must prove that $e$ is polarized.

If $n=2$ then $\Gamma$ is a generalized quadrangle. In this case $e$ is polarized by Proposition 2.6. Let $n>2$. For a point $x \in \mathcal{P}$, let $G_{x}$ be the stabilizer of $x$ in $G$ and let $K_{x}$ be the elementwise stabilizer of $\operatorname{Res}_{\Gamma}(x)$ in $G_{x}$. By Lemma 3.6, $G_{x} / K_{x}$ acts $B N$ transitively on $\operatorname{Res}_{\Gamma}(x)$ and $K_{x}$ is transitive on $\Gamma_{n}(x)$. In view of Theorem 3.1, in order to conclude that $e$ is polarized we only need to prove the following:

Lemma 4.1 The group $K_{x}$ acts trivially on $\mathrm{PG}\left(M_{i}(x)\right)$ for all $i=1,2, \ldots, n-1$.

Proof If $T_{i-1}(x)$ has codimension at most 1 in $T_{i}(x)$ there is nothing to prove. Suppose that $T_{i-1}(x)$ has codimension at least 2 in $T_{i}(x)$. We first prove the following:

(i) $p_{i}(C) \neq 0$ for every $C \in \mathcal{C}_{i}(x)$ and the mapping $p_{i}$ sending $C \in \mathcal{C}_{i}(x)$ to $p_{i}(C)$ is injective. 
As $G_{x}$ acts transitively on $\mathcal{C}_{i}(x)$, if $p_{i}(C)=0$ for some $C \in \mathcal{C}_{i}(x)$ then $p_{i}(C)=0$ for all $C \in \mathcal{C}_{i}(x)$. Hence $T_{i}(x)=T_{i-1}(x)$ by Proposition 2.7, contrary to our assumptions. So, $p_{i}(C) \neq 0$ for every $C \in \mathcal{C}_{i}(x)$. We now turn to the second part of (i). We know by Lemma 3.6 that $G_{x} / K_{x}$ acts $B N$-transitively on the projective geometry $\operatorname{Res}_{\Gamma}(x)$. The stabilizer of a member $C \in \mathcal{C}_{i}(x)$ in $G_{x} / K_{x}$ is a maximal parabolic subgroup of $G_{x} / K_{x}$. Hence the stabilizer $G_{x, C}$ of $C$ in $G_{x}$ is a maximal subgroup of $G_{x}$. Therefore $G_{x}$ cannot preserve any non-trivial proper partition of $\mathcal{C}_{i}(x)$. It follows that if $p_{i}\left(C_{1}\right)=p_{i}\left(C_{2}\right)$ for two distinct members $C_{1}, C_{2}$ of $\mathcal{C}_{i}(x)$, then $p_{i}\left(C_{1}\right)=p_{i}\left(C_{2}\right)$ for any two $C_{1}, C_{2} \in \mathcal{C}_{i}(x)$. Hence $T_{i-1}(x)$ is a hyperplane of $T_{i}(x)$, a contradiction with our assumptions. Therefore $p_{i}\left(C_{1}\right) \neq p_{i}\left(C_{2}\right)$ for any two distinct members $C_{1}, C_{2}$ of $\mathcal{C}_{i}(x)$. Claim (i) is proved.

We now put $S:=\left\{p_{i}(C)\right\}_{C \in \mathcal{C}_{i}(x)}$. By Proposition $2.7, S$ is a spanning set of points of $\mathrm{PG}\left(M_{i}(x)\right)$. If $\mathbb{F}=\mathbb{F}_{2}$ then $K_{x}$ fixes all points of $\operatorname{PG}\left(M_{i}(x)\right)$, as $S$ spans $\mathrm{PG}\left(M_{i}(x)\right)$ and $K_{x}$ fixes all points of $S$. In this case we are done.

Let $\mathbb{F} \neq \mathbb{F}_{2}$. We recall that $\mathcal{C}_{i}(x)$ is the point-set of the grassmannian $\mathcal{G}_{i}(x)$ of $\operatorname{Res}_{\Gamma}(x)$ and the collinearity graph of $\mathcal{G}_{i}(x)$ is connected. That graph induces a connected graph on $S$. By Lemma 3.4, in order to show that $K_{x}$ acts trivially on $\mathrm{PG}\left(M_{i}(x)\right)$ we only must prove the following:

(ii) Let $C_{1}$ and $C_{2}$ be distinct members of $\mathcal{C}_{i}(x)$, collinear in $\mathcal{G}_{i}(x)$. Then $K_{x}$ acts trivially on the line $L=\left\langle p_{i}\left(C_{1}\right), p_{i}\left(C_{2}\right)\right\rangle$.

If there is $C_{3} \in \mathcal{C}_{i}(x)$ such that $p_{i}\left(C_{3}\right) \in\left\langle p_{i}\left(C_{1}\right), p_{i}\left(C_{2}\right)\right\rangle$ and $p_{i}\left(C_{3}\right) \neq p_{i}\left(C_{1}\right)$, $p_{i}\left(C_{2}\right)$, then $K_{x}$ fixes three distinct points of $L$. Hence $K_{x}$ induces the identity mapping on $L$ by the following well-known fact.

Fact 4.2 Let $\mathbb{F}$ be a commutative division ring. Then the identity is the only element of $\operatorname{PGL}(2, \mathbb{F})$ that fixes three distinct points of $\mathrm{PG}(1, \mathbb{F})$.

(Recall that $\mathbb{F}$ is commutative by assumption and $K_{x}$ acts on $\mathrm{PG}(V)$ as a subgroup of $\operatorname{PGL}(V)$, by the definition of $G$.)

Suppose that $L \cap S=\left\{p_{i}\left(C_{1}\right), p_{i}\left(C_{2}\right)\right\}$. As $C_{1}, C_{2}$ are collinear in $\mathcal{G}_{i}(x), A:=$ $C_{1} \cap C_{2}$ is a member of $\mathcal{C}_{i-1}(x)$ and the convex closure $B:=\left[C_{1}, C_{2}\right]_{\Gamma}$ of $C_{1} \cup C_{2}$ belongs to $\mathcal{C}_{i+1}(x)$. The line of $\mathcal{G}_{i}(x)$ through $C_{1}$ and $C_{2}$ is the set $\mathcal{C}_{A, B}=\{C \in$ $\left.\mathcal{C}_{i}(x) \mid A \subset C \subset B\right\}$. Pick a point $y \in A \cap \Gamma_{i-1}(x)$ and a point $z \in B \cap \Gamma_{i+1}(x) \cap$ $\Gamma_{2}(y)$ and let $Q=[y, z]_{\Gamma}$ be the quad of $\Gamma$ on $y$ and $z$. Let $\mathcal{C}_{1}^{Q}(y)$ be the set of lines of $Q$ through $y$. The mapping $\gamma_{Q}$ sending $l \in \mathcal{C}_{1}^{Q}(y)$ to the convex closure $[l, A]_{\Gamma} \in$ $\mathcal{C}_{A, B}$ of $l \cup A$ is a bijection between $\mathcal{C}_{1}^{Q}(y)$ and $\mathcal{C}_{A, B}$. Its inverse map sends $C \in \mathcal{C}_{A, B}$ back to $C \cap Q$. Moreover, let $e_{Q}: Q \rightarrow\langle e(Q)\rangle$ be the embedding of $Q$ induced by $e$. Let $T_{1}^{Q}(y)$ be the tangent space of $e_{Q}$ of degree 1 at $e_{Q}(y)(=e(y))$ spanned by the lines $l \in \mathcal{C}_{1}^{Q}(y)$. Clearly, $T_{1}^{Q}(y)$ is a subspace of $T_{i}(x)$ and the inclusion mapping of $T_{1}^{Q}(y)$ into $T_{i}(x)$ induces a morphism $p_{Q}$ from $\operatorname{PG}\left(M_{1}^{Q}(y)\right)$ to $\operatorname{PG}\left(M_{i}(x)\right)$, where $M_{1}^{Q}(y):=T_{1}^{Q}(y) / e_{Q}(y)$. The morphism $p_{Q}$ agrees with $\gamma_{Q}$ on $\mathcal{C}_{1}^{Q}(y)$. Explicitly, if $p_{1}^{Q}$ is the mapping from $\mathcal{C}_{1}^{Q}(y)$ to $\operatorname{PG}\left(M_{1}^{Q}(y)\right)$ sending $l \in \mathcal{C}_{1}^{Q}(y)$ to the point $e(l) / e(y)$ of $\operatorname{PG}\left(M_{1}^{Q}(y)\right)$, then $p_{Q}\left(p_{1}^{Q}(l)\right)=p_{i}\left(\gamma_{Q}(l)\right)$ for every $l \in \mathcal{C}_{1}^{Q}(y)$. 
Put $S_{Q}:=\left\{p_{1}^{Q}(l) \mid l \in \mathcal{C}_{1}^{Q}(y)\right\}$, let $l_{1}, l_{2}$ be the lines of $\mathcal{C}_{1}^{Q}(y)$ that are sent by $\gamma_{Q}$ to $C_{1}$ and $C_{2}$ respectively, and let $L_{Q}$ be the line of $\operatorname{PG}\left(M_{1}^{Q}(y)\right)$ spanned by $p_{1}^{Q}\left(l_{1}\right)$ and $p_{1}^{Q}\left(l_{2}\right)$. Clearly, $L=p_{Q}\left(L_{Q}\right)$ and, since $L \cap S=\left\{p_{i}\left(C_{1}\right), p_{i}\left(C_{2}\right)\right\}$, we have $L_{Q} \cap S_{Q}=\left\{p_{1}^{Q}\left(l_{1}\right), p_{1}^{Q}\left(l_{2}\right)\right\}$. By Lemma 3.3, there are lines $l_{3}, l_{4} \in \mathcal{C}_{1}^{Q}(y)$ such that the line $L_{Q}^{\prime}=\left\langle p_{1}^{Q}\left(l_{3}\right), p_{1}^{Q}\left(l_{4}\right)\right\rangle$ of $\operatorname{PG}\left(M_{1}^{Q}(y)\right)$ meets $L_{Q}$ in a point different from any of $p_{1}^{Q}\left(l_{1}\right), p_{1}^{Q}\left(l_{2}\right), p_{1}^{Q}\left(l_{3}\right)$ or $p_{1}^{Q}\left(l_{4}\right)$. For $j=3,4$, put $C_{j}=\gamma_{Q}\left(l_{j}\right)$ and let $L^{\prime}$ be the line of $\operatorname{PG}\left(M_{i}(x)\right)$ spanned by $p_{i}\left(C_{3}\right)$ and $p_{i}\left(C_{4}\right)$. Then $L^{\prime}=p_{Q}\left(L_{Q}^{\prime}\right)$ and $L \cap L^{\prime}$ is a point different from any of $p_{i}\left(C_{1}\right), p_{i}\left(C_{2}\right), p_{i}\left(C_{3}\right)$ or $p_{i}\left(C_{4}\right)$. Indeed, if otherwise, then we have $p_{i}\left(C_{3}\right), p_{i}\left(C_{4}\right) \in L \cap S$, which contradicts the assumption that $L \cap S=\left\{p_{i}\left(C_{1}\right), p_{i}\left(C_{2}\right)\right\}$.

We can now finish the proof of (ii). As $K_{x}$ fixes each of $C_{1}, C_{2}, C_{3}$ and $C_{4}$, it also fixes the point $L \cap L^{\prime}$. So, $K_{x}$ fixes three distinct points of $L$. Hence it acts trivially on $L$, by Fact 4.2 and since $K_{x} \subseteq G \leq \operatorname{Aut}(\Gamma)_{\text {lin }}$ acts on $\mathrm{PG}(V)$ as a subgroup of $\operatorname{PGL}(V)$.

Remark In Lemma 4.1 we could replace $K_{x}$ by $U_{x}$. Then that lemma follows immediately for any embedding $V$ of $\Gamma$ on which $U_{x}$ is known to act unipotently.

\subsection{Proof of Theorem 1.2}

Let $\Gamma=(\mathcal{P}, \mathcal{L})$ be an embeddable classical dual polar space of rank $n \geq 2$ and let $G=\operatorname{Aut}(\Gamma)_{0}$. The group $G$ acts $B N$-transitively on the building $\Delta=\Delta(\Gamma)$. Let $e: \Gamma \rightarrow \Sigma$ be a $G$-homogeneous polarized embedding of $\Gamma$ and let $U$ be a $G$-invariant proper subspace of $\Sigma$.

Lemma 4.3 The subspace $U$ defines a $G$-homogeneous quotient of e.

Proof Clearly, if $U$ defines a quotient $e / U$ of $e$ then $e / U$ is $G$-homogeneous, since $U$ is $G$-invariant. So, we only must prove that $U$ defines a quotient of $e$, namely it satisfies conditions (Q1), (Q2) of Subsection 2.2. If $e(x) \in U$ for some point $x$, then $U$ contains $e(\mathcal{P})$ by the transitivity of $G$ on $\mathcal{P}$. Hence $U=\Sigma$. This contradicts the assumption that $U \neq \Sigma$. Therefore $U \cap e(\mathcal{P})=\emptyset$, namely $U$ satisfies (Q1).

For $d=1, \ldots, n$, let $\mathcal{G}_{d}$ be the graph defined on $\mathcal{P}$ by the relation 'being at distance $d$ '. We shall prove that $\mathcal{G}_{d}$ is connected. First, let $x, y$ be two distinct collinear points of $\Gamma$. By Lemma 2.2, $\Gamma_{d}(x) \cap \Gamma_{d}(y) \neq \emptyset$. Clearly, each of $\{x\} \cup \Gamma_{d}(x)$ and $\{y\} \cup \Gamma_{d}(y)$ is contained in a connected component of $\mathcal{G}_{d}$. Since $\Gamma_{d}(x) \cap \Gamma_{d}(y) \neq \emptyset$, $\{x, y\} \cup \Gamma_{d}(x) \cup \Gamma_{d}(y)$ is contained in a connected component of $\mathcal{G}_{d}$. In other words, any two collinear points of $\Gamma$ belong to the same connected component of $\mathcal{G}_{d}$. Hence $\mathcal{G}_{d}$ is connected, since the collinearity graph of $\Gamma$ is connected.

Suppose now that $\langle e(u), U\rangle=\langle e(v), U\rangle$ for two distinct points $u, v \in \mathcal{P}$. Let $d=d(u, v)$. The graph $\mathcal{G}_{d}$ is connected. Moreover, $G$ acts transitively on the set of ordered edges of $\mathcal{G}_{d}$, since it is $B N$-transitive on $\Delta$. Therefore the equality $\langle e(u), U\rangle=\langle e(v), U\rangle$ implies that $\langle e(x), U\rangle=\langle e(y), U\rangle$ for any two points $x, y \in \mathcal{P}$. As $e(\mathcal{P})$ spans $\Sigma$, it follows that $U$ is a hyperplane of $\Sigma$. However if so, then 
$U \cap e(l) \neq \emptyset$ for every line $l$ of $\Gamma$, contrary to the fact that $U \cap e(\mathcal{P})=\emptyset$. Therefore $\langle e(x), U\rangle \neq\langle e(y), U\rangle$ for any two distinct points $x, y \in \mathcal{P}$. Consequently, $U$ satisfies (Q2).

Theorem 4.4 With $\Gamma, G$, e and $U$ as above, suppose moreover that $\Gamma$ is defined over a commutative division ring. Then $U$ is contained in the nucleus $R_{e}$ of $e$.

Proof By Lemma 4.3, $U$ defines a $G$-homogeneous quotient $e / U$ of $e$. As the underlying division ring of $\Gamma$ is assumed to be commutative, we can apply Theorem 1.1, thus concluding that $e / U$ is polarized. Therefore $U \subseteq R_{e}$.

Theorem 1.2 is a special case of the above.

\section{References}

1. Adamovich, A.M.: The submodule lattice of Weyl modules for symplectic groups with fundamental highest weights. Mosc. Univ. Math. Bull. 41, 6-9 (1986)

2. Blok, R.J., Brouwer, A.E.: Spanning point-line geometries in buildings of spherical type. J. Geom. 62, 26-35 (1998)

3. Blok, R.J., Cardinali, I., De Bruyn, B.: On the nucleus of the Grassmann embedding of the symplectic dual polar space $\operatorname{DSp}(2 n, \mathbb{F}), \operatorname{char}(\mathbb{F})=2$. Eur. J. Comb. 30, 468-472 (2009)

4. Blok, R.J., Cooperstein, B.N.: The generating rank of the unitary and symplectic grassmannians. Preprint, July 2006

5. Blokhuis, A., Brouwer, A.E.: The universal embedding dimension of the binary symplectic dual polar space. Discrete Math. 264, 3-11 (2003)

6. Buekenhout, F., Cameron, P.J.: Projective and affine geometry over division rings. In: Buekenhout, F. (ed.) Handbook of Incidence Geometry, pp. 27-62. Elsevier, Amsterdam (1995)

7. Buekenhout, F., Cohen, A.M.: Diagram Geometry, in preparation; preliminary draft available at http://www.win.tue.nl/ amc/buek

8. Buekenhout, F., Lefèvre, C.: Generalized quadrangles in projective spaces. Arch. Math. 25, 540-552 (1974)

9. Cardinali, I., De Bruyn, B.: The structure of full polarized embeddings of symplectic and Hermitian dual polar spaces. Adv. Geom. 8, 111-137 (2008)

10. Cardinali, I., De Bruyn, B., Pasini, A.: Minimal full polarized embeddings of dual polar spaces. J. Algebr. Comb. 25, 7-23 (2007)

11. Cardinali, I., De Bruyn, B., Pasini, A.: On the simple connectedness of hyperplane complements in dual polar spaces. Discrete Math. 309, 294-303 (2009)

12. Cardinali, I., Lunardon, G.: A geometric description of the spin-embedding of symplectic dual polar spaces of rank 3. J. Comb. Theory Ser. A 115, 1056-1064 (2008)

13. Cooperstein, B.N.: On the generation of dual polar spaces of unitary type over finite fields. Eur. J. Comb. 18, 849-856 (1997)

14. Cooperstein, B.N.: On the generation of dual polar spaces of symplectic type over finite fields. J. Comb. Theory Ser. A 83, 221-232 (1998)

15. Cooperstein, B.N., Shult, E.E.: Geometric hyperplanes of embeddable Lie incidence geometries. In: Hirschfeld, J.W.P., et al. (eds.) Advances in Finite Geometries and Designs, pp. 81-91. Oxford University Press, Oxford (1991)

16. Cooperstein, B.N., Shult, E.E.: Frames and bases of Lie incidence geometries. J. Geom. 60, 17-46 (1997)

17. Cooperstein, B.N., Shult, E.E.: A note on embedding and generating dual polar spaces. Adv. Geom. 1, 37-48 (2001)

18. De Bruyn, B.: Near Polygons. Frontiers in Mathematics. Birkhäuser, Basel (2006)

19. De Bruyn, B.: A decomposition of the natural embedding spaces for the symplectic dual polar spaces. Linear Algebra Appl. 426, 462-477 (2007) 
20. De Bruyn, B.: On the Grassmann-embeddings of the hermitian dual polar spaces. Linear Multilinear Algebra 56, 665-677 (2008)

21. De Bruyn, B.: The structure of the spin-embeddings of dual polar spaces and related geometries. Eur. J. Comb. 29, 1242-1256 (2008)

22. De Bruyn, B.: A note on the spin-embedding of the dual polar space $D Q^{-}(2 n+1, \mathbb{K})$. Ars Comb. (to appear)

23. De Bruyn, B.: Dual embeddings of dense near polygons. Ars Comb. (to appear)

24. De Bruyn, B.: On the Grassmann modules for the symplectic and unitary groups. Preprint, December 2007

25. De Bruyn, B., Pasini, A.: Generating symplectic and Hermitian dual polar spaces over arbitrary fields nonisomorphic to $\mathbb{F}_{2}$. Electron. J. Comb. 14 (2007), \#R54, 17pp

26. De Bruyn, B., Pasini, A.: Minimal scattered sets and polarized embeddings of dual polar spaces. Eur. J. Comb. 28, 1890-1909 (2007)

27. Dienst, K.J.: Verallgemeinerte Vierecke in projektiven Räumen. Arch. Math. 35, 177-186 (1980)

28. Johnson, P.M.: Semiquadratic sets and embedded polar spaces. J. Geom. 64(1-2), 102-127 (1999)

29. Kasikova, A., Shult, E.E.: Absolute embeddings of point-line geometries. J. Algebra 238, 265-291 (2001)

30. Li, P.: On the universal embedding of the $S p_{2 n}(2)$ dual polar space. J. Comb. Theory Ser. A 94, 100-117 (2001)

31. Li, P.: On the universal embedding of the $U_{2 n}(2)$ dual polar space. J. Comb. Theory Ser. A 98, 235252 (2002)

32. Pasini, A., Van Maldeghem, H.: Some constructions and embeddings of the tilde geometry. Note Mat. 21(2), 1-33 (2002/2003)

33. Premet, A.A., Suprunenko, I.D.: The Weyl modules and the irreducible representations of the symplectic group with the fundamental highest weights. Commun. Algebra 11, 1309-1342 (1983)

34. Ronan, M.A.: Embeddings and hyperplanes of discrete geometries. Eur. J. Comb. 8, 179-185 (1987)

35. Ronan, M.A.: Lectures on Buildings. Perspectives in Mathematics, vol. 7. Academic Press, Boston (1989)

36. Shult, E.E.: On Veldkamp lines. Bull. Belg. Math. Soc. Simon Stevin 4, 299-316 (1997)

37. Taylor, E., Donald, X.: The Geometry of the Classical Groups. Sigma Series in Pure Mathematics, vol. 9. Heldermann, Berlin (1992)

38. Tits, J.: Buildings of Spherical Type and Finite BN-pairs. Lecture Notes in Mathematics, vol. 386. Springer, Berlin (1974)

39. Tits, J., Weiss, R.M.: Moufang Polygons. Springer, Berlin (2002)

40. Weiss, R.M.: The Structure of Spherical Buildings. Princeton University Press, Princeton (2003)

41. Wells, A.: Universal projective embeddings of the Grassmannian, half spinor, and dual orthogonal geometries. Q. J. Math. Oxf. 34, 375-386 (1983) 